\title{
Infants Respond to Robot's Need for Assistance in Pursuing Action-based Goals
}

\author{
Elena Kokkoni \\ Amanda J. Arnold \\ elena.kokkoni@ucr.edu \\ amandaa@ucr.edu \\ University of California, Riverside \\ Riverside, CA, USA
}

\author{
Kleio Baxevani \\ Herbert G. Tanner \\ kleiobax@udel.edu \\ btanner@udel.edu \\ University of Delaware \\ Newark, DE, USA
}

\begin{abstract}
Instrumental helping has been reported in infants toward other humans but not toward robots. Providing infants with opportunities for action-based assistance to robots might lead to more efficient infant-robot interactions. This paper presents preliminary findings on infants' spontaneous instrumental helping to robots exhibiting motion challenges, and proposes a novel decision-making model for infant-robot interaction that encompasses instrumental helping in its parameters; both in the context of pediatric rehabilitation. Six infants were engaged in a chasing game with a wheeled robot with the goal to follow the robot and ascend an inclined platform (8 sessions, 4 weeks). After infants' instrumental helping toward the robot was identified, a decision tree model was created to evaluate a set of annotated variables as potential predictors to the observed behavior. Next, a Markovian model for robot control was developed where these predictors were used as parameters to promote, in turn, action-based goals for the infants.
\end{abstract}

\section{CCS CONCEPTS}

- Human-centered computing $\rightarrow$ Human computer interaction (HCI); Collaborative and social computing; • Computer systems organization $\rightarrow$ Robotics.

\section{KEYWORDS}

human-robot interaction, prosocial, decision tree, Markov process

ACM Reference Format:

Elena Kokkoni, Amanda J. Arnold, Kleio Baxevani, and Herbert G. Tanner. 2021. Infants Respond to Robot's Need for Assistance in Pursuing Actionbased Goals. In Companion of the 2021 ACM/IEEE International Conference on Human-Robot Interaction (HRI '21 Companion), March 8-11, 2021, Boulder, CO, USA. ACM, New York, NY, USA, 5 pages. https://doi.org/10.1145/3434074. 3447126

\section{INTRODUCTION}

From a very young age, humans are capable of perceiving the need for assistance and assisting other humans in their pursuit of actionbased goals (i.e. instrumental helping) [35]. As early as in the second

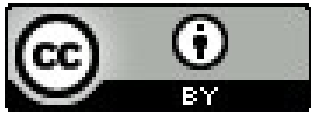

This work is licensed under a Creative Commons Attribution International 4.0 License. HRI '21 Companion, March 8-11, 2021, Boulder, CO, USA. (C) 2021 Copyright held by the owner/author(s).

ACM ISBN 978-1-4503-8290-8/21/03. https://doi.org/10.1145/3434074.3447126 year of life, infants can pass objects to adults that are out of the latter's reach, remove physical obstacles for them, and use pointing gestures to indicate object locations $[23,36]$. In the first year of life, they can assist adults in chores and their own self-care tasks at home [11]. More fascinating is that infants engage in these actions spontaneously; either for no apparent tangible benefit or sometimes to receive social praise, whereas receiving a material award may reduce the probability for further assistance [37]. On the other hand, prior observation of prosocial behavior in adults increases the number of instrumental helping actions by infants [34]. Yet, we neither know if this prosocial behavior exists in infants toward machines, nor the circumstances (e.g., reward type needed) under which this behavior could be elicited. Recently, there has been an increased interest in infant-robot interaction [4, 9, 17, 29] for the potential benefits it may offer if applied in the early stages of human development. Therefore, assessing infants' prosocial behavior toward robots is essential so as to create mathematical models in infant-robot interaction, that can be used to elicit this behavior for various early life applications (e.g., rehabilitation, education, etc.).

In our context, instrumental helping refers to assisting a robot when the latter is unable to reach its goal on its own. One can contrast this to the traditional role of assistive robots in which they help the human they interact with. Instrumental helping toward robots, however, can lead to more efficient, symbiotic human-robot interactions [32]. Limited work with older children supports their capacity to assist robots in handover tasks [1,24, 25, 39]. Children in kindergarten are capable of passing objects to a humanoid robot that drops those in front of them [39]. Three-year old children are even capable of attributing goals to a robot and understanding the latter's need for help by assisting only in cases when the latter drops the object unintentionally [25]. Yet to our knowledge, no studies have examined if instrumental helping toward robots can be exhibited even earlier in life, in infancy.

Realizing natural and efficient human-robot interaction (HRI) can be facilitated by dynamic and highly-adaptive automated decisionmaking models (such as $[14,26]$ ). In infant-robot social interaction, developing such models is even more challenging, due to unique population characteristics, such as the rapidly-changing learning mechanisms and the high levels of infants' curiosity and distraction when they explore their environment. Such models and algorithms, therefore, need to be quickly adaptive and personalized in applications that involve young populations. Few mathematical models to address these challenges have been proposed [40, 41]. We build upon these models to develop a new model for infant-robot interaction that contains the behavior of interest in this paper, instrumental 
helping; the foundation of which was based on first-time real observations of a group of infants spontaneously assisting robots while participating in a complex motor task.

This paper sheds some light into the underlying factors that facilitate the emergence of infants' assistive actions toward robots, and proposes a new decision-making model for robot control to artificially elicit this prosocial behavior in infants toward robots. Infant-robot interaction data were collected in a motor rehabilitation paradigm, and thus, the proposed work is within this context. We aimed to: (a) quantify infants' action-based helping behavior toward robots when the latter exhibited challenges in completing the task, (b) examine the factors under which infant-to-robot assistance has greater probability to be observed, and (c) outline ways these insights can inform the construction of mathematical infant-robot prosocial interaction models that capture this early behavior.

\section{METHODS}

Six pre-walking infants ( $M=12.7$ months of age, one with Down syndrome [DS]) participated in eight 1-hour sessions over four weeks. In each session, infants engaged in motor tasks while interacting with socially assistive robots. This paper focuses specifically on a platform ascending task (PAT), which provided more frequent and consistent opportunities for infants to interact prosocially with the robotic play companion. In PAT, infants were encouraged to climb an inclined platform by following a small wheeled robot (Dash $^{\mathrm{TM}}$ Wonder Workshop) (Fig. 1). Both companions started the task at the bottom of the inclined platform. The robot started ascending first, in an effort to entice the infant into chasing it. At the top of the platform, the infant would receive reward through social praise by adults, manipulation of Dash, and/or Dash making sounds and flashing lights. In half of the trials, infants used the assistance of a body weight support system (BWSS) that provided mechanical support to ease moving [15, 16, 18, 31]. Sessions were recorded (@15 frames/sec) via a network of five cameras (KINECT ${ }^{\mathrm{TM}}$ Microsoft).

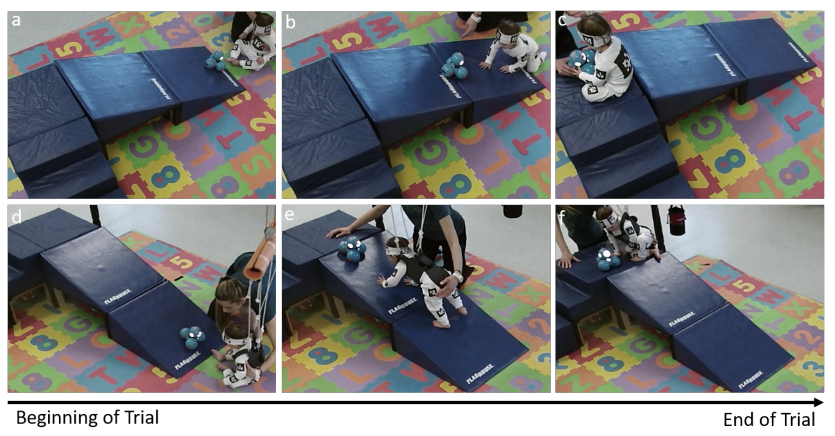

Figure 1: Snapshots of an infant participating in PAT trials without (a-c) and with (d-f) the assistance from the BWSS.

PAT was designed to exploit infant-robot social interactions to elicit and assess infants' motor response in a natural, play-type scenario. As is typical in such scenarios involving young children, PAT trials did not always evolve according to plan; e.g., infants may not follow the robot, or Dash may slip and/or fall over to the side. To maintain the interaction between the two agents and see through task completion, the researcher would often physically assist the robot facing a challenge (i.e., put it back on course). The random nature of the robot's challenges, as well as that of researcher's assistive interventions in the robot's motion, presented varying (unplanned) opportunities (within each session, across sessions, and across subjects) to the infants for expression of spontaneous instrumental helping toward the robot. Nonetheless, these opportunities proved sufficient for the infants to exhibit this behavior at a frequency that attracted the researchers' attention, and triggered them to pose questions related to the emergence of this behavior in multi-factorial environments resembling natural real-world situations [20], and at no cost of simplifying a complex phenomenon that would undermine ecological validity $[22,33]$.

This paper's approach involves three steps. First, variables of interest were annotated from recordings of 117 PAT trials to quantify if and when infants assisted the robot, and describe the conditions under which infant-to-robot assistance occurred (Table 1).

Table 1: Annotated Variables and Descriptions

Robot Faces Challenge (Y/N): Cannot ascend, falls off or slides back down on platform, decelerates and/or remains stationary.

Robot's Position Within Infant's Reach (Y/N): Robot is within infant arm's reaching distance at any point during its challenge. Infant Looks at Robot (Y/N): Infant shifts visual attention toward robot at any point during the latter's challenge.

Infant Assists Robot (Y/N): Infant gently pushes robot into ascending at any point during the latter's challenge.

Latency to Infant's Assistance (No. of Frames): Time elapsed from 1st frame robot entered infant's reaching space or faced challenge (if within that space), to 1st frame assistance occurred. Location on Platform Assistance Occurred (B/M/T)]: Bottom: on the ground and up to $25 \%$ of platform length, Middle: at any point between $25 \%$ and $75 \%$ of platform length, Top: over $75 \%$ of platform length and on the top flat surface. Mechanical Support to Infant's Body (Y/N): Infant uses the assistance from the body weight support system during ascend. Study Session Assistance Occurred (1st/2nd half): 1-4/5-8.

Then, decision trees were formed from the annotated dataset to get an insight on how these variables interacted to result in the behavior of interest. To create those, two Chi-square Automatic Interaction Detection (CHAID) data mining methods for categorical variables were applied (simple and exhaustive). CHAID detects associations between predictors and the outcome variable to identify those factors that predict best the likelihood of the outcome variable to occur $[6,10,13]$. The analysis was performed in SPSS v.24 (IBM Statistics, Armonk, NY) without restrictions (considering any number of cases in the nodes). Chi-square analysis was used to determine node splitting and category merging using the likelihood-ratio method, considered more appropriate than Pearson's for small data sets. The alpha level for all statistical tests was adjusted for multiple comparisons using the Bonferroni correction.

Lastly, information obtained from previous steps was used to construct a mathematical HRI model that captures aspects of infantrobot prosocial interaction. The underlying robot control laws that 
are represented abstractly in this model, are discussed. A typical model for HRI is a Markov decision process (MDP) [14, 26], where optimal decision-making policies can be derived for maximizing the utility the robot extracts toward defined goals. In the instantiations of MDP models developed for infant-robot interaction [17, 40, 42], the transition probabilities are tuned based on observational data. Different configurations or modes that the combined infant-robot system can find itself into are encoded, and transitions are labeled based on controlled robot actions designed to elicit particular human motor responses. This paper extends prior work and reveals new ways for the robot to engage with the infant by including and eliciting prosocial behavior in its parameters.

\section{RESULTS}

Assistance to Robot. Infants had 214 opportunities to assist the robot facing a motion challenge. They looked at the robot in most of these instances $(N=163,76 \%)$; however, the robot was within infants' reach only in half of the instances $(N=106,50 \%)$. Researchers assisted in $74 \%$ of the 214 instances $(N=159)$. Infants looked at the robot in $79 \%$ of these instances $(N=126)$. In the remaining 55 cases, the robot was either assisted by the infants $(N=18,33 \%)$ or not at all $(N=37,67 \%)$. In the cases it was assisted, assistance was provided within a second (median Latency $=10$ frames).

Factors Linked to Assistance. Both CHAID analyses (considering five annotated predictors along with DS diagnosis) were conducted on the 55 remaining opportunities infants had to assist the robot. The first predictor to infant-to-robot assistance was distance between infant and robot $\left(p=0.000, \chi^{2}=29.165\right)$, followed by infant looking at the robot $\left(p=0.014, \chi^{2}=5.999\right)$, and then by location of assistance ( $p=0.035, \chi^{2}=4.460$ ) (Fig.2). Predictions in this model are likely to be wrong in roughly one in ten cases (risk value $=0.109$ ).

Model for Robot Decision Making. The proposed model builds upon a previous MDP model designed for games of chase, and is a parallel composition of abstract transition systems for the infant and robot, respectively $[19,40]$. The new insights obtained from the analysis of action-based assistance in this paper allow for refinement of the MDP model when applied to PAT cases, through the introduction of new states and transitions. The more sophisticated MDP model (cf. $[19,40])$ created for the PAT scenario includes four actions of the robot, getting values from the set $\{f, b, s, h\}: f$ for moving forward; $b$ for moving backwards; $s$ for staying still; and $h$ for pretending to struggle. The two possible states of the infant are denoted $G$ and $N$, representing the infant responding or not, respectively, to a robot action. The three predictors of the decision tree in Fig. 2 are also states in this model: if the robot is within reach from the infant or not $\{D Y, D N\}$; if the infant is looking at the robot or not $\{L Y, L N\}$; and the location of the robot (bottom or top) $\{B, T\}$. States labeled with $\{?\}$ signify that the particular predictor state component can have either of the two possible values.

The new MDP model for PAT, extended with the new states and transitions, as dictated by the observations and statistical analysis of this paper, is shown in its prior form in Fig. 3. This MDP model is conservatively initialized essentially assuming that robot actions have no effect on infant motor behavior-thus not all the parts of the MDP are connected. However, as new instances of the behaviors of interest are observed, a learning algorithm $[40,42]$ adds new transitions and tunes their probabilities. This process will continue until our model converges to a hypothesized true probabilistic model of this personalized HRI.

In addition to the states and transitions in the MDP model, the factors that are found likely to trigger the assistive behavior of interest also inform the design of the robot controllers behind each robot action in the set $\{f, b, s, h\}$ : for instance, for action $h$, we now know what the robot should need to ensure before pretending to struggle as a means of inciting infant assistance. The analytic design of these control laws is beyond the scope of this paper.

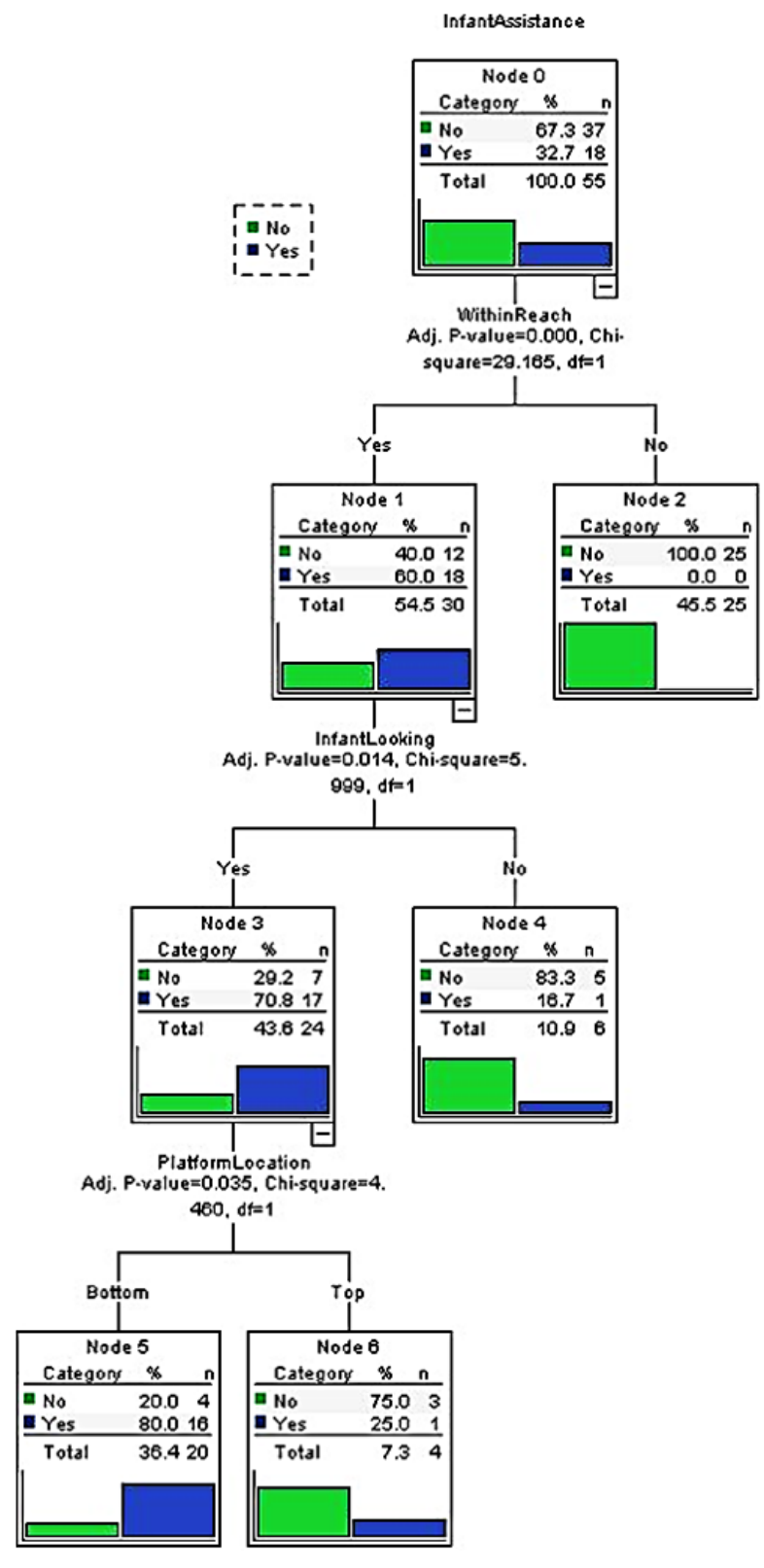

Figure 2: Decision tree showing the independent variable (predictor) with the strongest interaction with the dependent variable (assistance) at each step of CHAID analysis. 

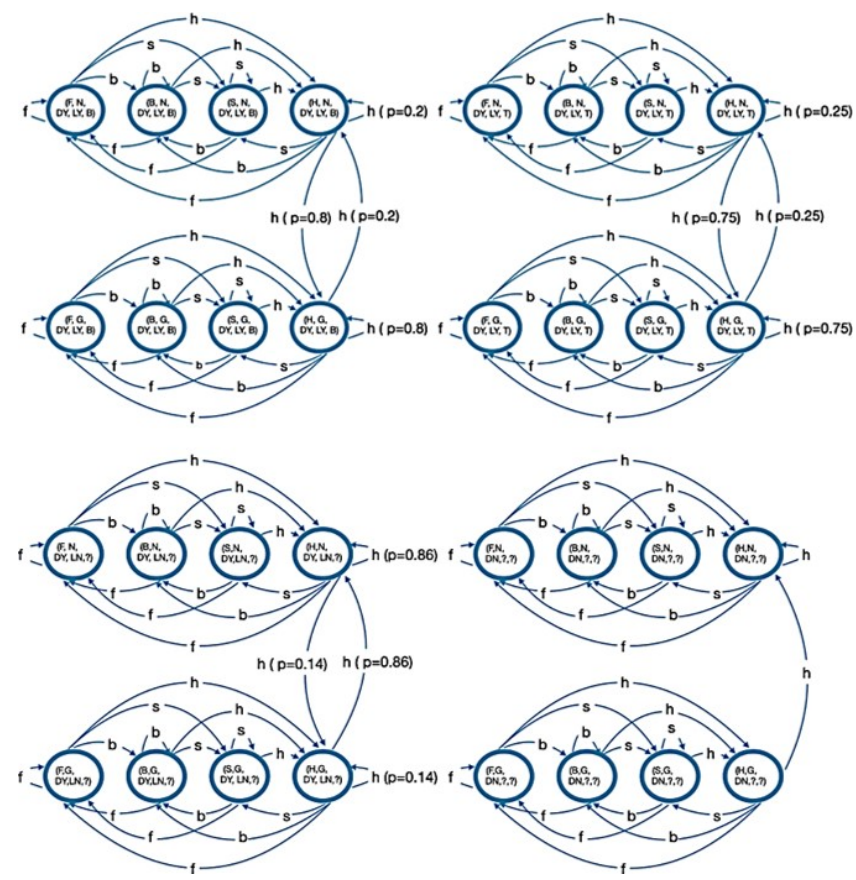

Figure 3: MDP model for infant-robot prosocial interaction.

\section{DISCUSSION}

This paper provides, for the first time, support for infants' instrumental helping toward robots, and describes the underlying conditions surrounding the emergence of infant-to-robot assistance when the latter exhibits motion challenges during participation in a complex, but ecologically-valid, motor task. These results are important as they lay the foundation for building infant-robot prosocial interaction environments and models that can be utilized in various applications early in life, an example of which is described here.

In human interactions, there is an ongoing debate on the nature of this prosocial behavior. Some researchers suggest that it indicates infants' affiliative motives [27,28], whereas others suggest that it is just the result of a simple desire to carry out an incomplete action [5]. It has also been proposed that early helping behavior is based on infants' understanding of others' needs [7, 38]. More recent work, however, suggests that the emergence of this behavior may be more complex; it can be the output of a developmental system characterized by the infants' ability to understand the needs of others along with their social and motor capabilities [20]. Although our preliminary results can not accurately answer this question with respect to infant-to-robot assistance at this time, they open the ground for a similar debate in infant-robot interaction and the examination of the nature of this behavior in the future.

Finding that infants are capable of instrumental helping to robots was surprising as (a) the neurotypical infants that participated in this study were younger than 12 months old (infants begin to reliably help others around 16 months of age [21]), and (b) a nonanthropomorphic agent was on the receiving end of the assistance (compared to humanoids and robotic arms previously used in childrobot prosocial interaction paradigms [1, 24, 25, 39]). Nevertheless, the fact that the infant with DS (24 months of age) showed higher assistance rates (11 assists) than the neurotypical peers ( 7 assists), supports previous work on reliable demonstration of helping behavior toward humans during the second year of life of infants with [30] and without DS [21]. Further testing with slightly older neurotypical infants will examine if infant-to-robot assistance rates increase during the second year of life.

Regarding the underlying conditions surrounding infant-to-robot assistance, it came as no surprise that close distance between infant and robot was the first predictor for helping actions. Proximity has been suggested to be an important factor for interactions of crawling infants (i.e., like the participants of this study) displaying a tendency to interact with nearby objects $[8,12]$ and to help less in out-of-reach tasks [21]. The second predictor to instrumental helping, looking at the robot facing a motion challenge, suggests that infants gather information regarding the robots' need of assistance through visual cues (e.g., robot getting stuck on the platform) and through observation of the researcher's assistance toward the robot [34]. Infants as young as nine months of age are capable of understanding others' needs in looking paradigms-this understanding appears even before they engage in helping behaviors themselves [21]. Lastly, the location where most assisting actions occurred was the bottom of the platform, possibly due to the greater number in opportunities for assistance within reaching distance in the beginning of the chasing game.

Other variables deemed important to instrumental helping were not identified as predictors. For example, time in study did not seem to affect helping; indeed observations of this behavior were spread out, starting as soon as the second session. Another variable was the mechanical support to the infants' bodies, which was used to alleviate the effects from gravity on movement $[16,17]$. We hypothesized that the increase in motor competency would allow infants to use cognitive and attentional resources to evaluate the robot's motion challenge and assist it [2,3]. Given these expectations, it was surprising that the above variable were not predictors.

A number of limitations may warrant future consideration. Although the ecological nature of the experimental paradigm is a strength, it did lead to some infants having more opportunities for assistance than others. Another limitation is the small amount of observations in this study. Although data were collected over several sessions $(\mathrm{N}=48)$, a larger sample size may produce different results. Such limitations are common in human experimental paradigms but can impact models of infant-robot prosocial interaction, such as the one presented here. The implication is that naive methods for updating the transition probabilities (e.g. maximum likelihood estimation) produce a poor MDP model parameterization [40]. A remedy to issues arising from small data sets such as this one can be data smoothing, which has been previously proposed in HRI $[40,42]$, and can help overcome the scarcity of learning data in infant-robot prosocial interaction applications.

\section{ACKNOWLEDGMENTS}

This work was supported by NIH 5R01HD87133. We thank the families for participation and lab researchers for their assistance. 


\section{REFERENCES}

[1] Tanya N. Beran, Alejandro Ramirez-Serrano, Roman Kuzyk, Sarah Nugent, and Meghann Fior. 2011. Would Children Help a Robot in Need? International fournal of Social Robotics 3, 1 (2011), 83-93. https://doi.org/10.1007/s12369-010-0074-7

[2] Sarah Berger. 2010. Locomotor Expertise Predicts Infants' Perseverative Errors Developmental Psychology 46, 2 (2010), 326-336.

[3] Sarah E. Berger, Regina T. Harbourne, and Melissa N. Horger. 2018. Cognition-Action Trade-Offs Reflect Organization of Attention in Infancy. In Advances in Child Development and Behavior. https://doi.org/10.1016/bs.acdb.2017.11.001

[4] De Aira Bryant, Janelle Boyd, Jgenisius Harris, Michelle Smith, Sergio Garcíavergara, and Yu-ping Chen. 2017. An Infant Smart-Mobile System to Encourage Kicking Movements in Infants At-Risk of Cerebral Palsy. In IEEE Workshop on Advanced Robotics and Its Social Impacts (ARSO). 1-5.

[5] Jeremy I.M. Carpendale, Viktoria A. Kettner, and Karyn N. Audet. 2015. On the nature of toddlers' helping: Helping or interest in others' activity? Social Development 24,2 (2015), 357-366. https://doi.org/10.1111/sode. 12094

[6] Fong Chan, Gladys Cheing, Jacob Yui Chung Chan, David A. Rosenthal, and Julie Chronister. 2006. Predicting employment outcomes of rehabilitation clients with orthopedic disabilities: A CHAID analysis. Disability and Rehabilitation 28, 5 (2006), 257-270. https://doi.org/10.1080/09638280500158307

[7] William Damon and Nancy Eisenberg. 1998. Handbook of child psychology, 5th ed.: Vol 3. Social, emotional, and personality development. US: John Wey \& Sons In-US: John Wey \& Sons In pages.

[8] Jill A. Dosso and J. Paul Boudreau. 2014. Crawling and walking infants encounter objects differently in a multi-target environment. Experimental Brain Research 232, 10 (2014), 3047-3054. https://doi.org/10.1007/s00221-014-3984-z

[9] Naomi Fitter, Rebecca Funke, Jose Carlos Pulido Pascual, Lauren E. Eisenman, Weiyang Deng, Marcelo R Rosales, Nina Bradley, Barbara Sargent, Beth Smith, and Maja Mataric. 2019. Socially Assistive Infant-Robot Interaction: Using Robots to Encourage Infant Leg-Motion Training. IEEE Robotics \& Automation Magazine (2019), 1-13. https://doi.org/10.1109/MRA.2019.2905644

[10] Rene Gonzalez, David A. Rosenthal, and Jeong Han Kim. 2011. Predicting vocational rehabilitation outcomes of young adults with specific learning disabilities: Transitioning from school to work. Fournal of Vocational Rehabilitation 34, 3 (2011), 163-172. https://doi.org/10.3233/JVR-2011-0544

[11] Stuart I. Hammond, Elizabeth Al-Jbouri, Victoria Edwards, and Laura E. Feltham 2017. Infant helping in the first year of life: Parents' recollection of infants' earliest prosocial behaviors. Infant Behavior and Development 47 (2017), 54-57. https://doi.org/10.1016/j.infbeh.2017.02.004

[12] Lana B. Karasik, Catherine S. Tamis-LeMonda, and Karen E. Adolph. 2014. Tran sition From Crawling to Walking and Infants' Actions With Objects and People. Child Development 82, 4 (2014), 1199-1209.

[13] G.V. Kass. 1980. An Exploratory Technique for Investigating Large Quantities of Categorical Data. Journal of the Royal Statistical Society. Series C (Applied Statistics) 29, 2 (1980), 119-127.

[14] Simon Keizer, Mary E Foster, Oliver J Lemon, Andre Gaschler, and Giuliani Manuel. 2013. Training and evaluation of an MDP model for social multi-user human-robot interaction. Proceedings of the SIGDIAL 2013 Conference (2013), 223--232.

[15] Elena Kokkoni and James C Galloway. 2019. User-centred assistive technology assessment of a portable open-area body weight support system for in-home use. Disability and Rehabilitation: Assistive Technology (2019), 1-8. https://doi.org/10 1080/17483107.2019.1683236

[16] Elena Kokkoni, Samuel W. Logan, Tracy Stoner, Terri Peffley, and James C. Galloway. 2018. Use of an In-home Body Weight Support System by a Child with Spina Bifida. Pediatric Physical Therapy 30 (2018), E1-E6.

[17] Elena Kokkoni, Effrosyni Mavroudi, Ashkan Zehfroosh, James C Galloway, Renè Vidal, Jeffrey Heinz, and Herbert G Tanner. 2020. GEARing smart environments for pediatric motor rehabilitation. fournal of NeuroEngineering and Rehabilitation 17, 1 (2020), 16. https://doi.org/10.1186/s12984-020-0647-0

[18] Elena Kokkoni, Tracy Stoner, and James C Galloway. 2020. In-Home Mobility Training With a Portable Body Weight Support System of an Infant With Down Syndrome. Pediatric Physical Therapy 32, 4 (2020), E76-E82. https://doi.org/10 1097/PEP.0000000000000752

[19] Elena Kokkoni, Ashkan Zehfroosh, Prasanna Kannappan, Effrosyni Mavroudi, James C Galloway, Jeffrey Heinz, Rene Vidaly, and Herbert G Tanner. 2017. Principles for Building "Smart" Learning Environments in Pediatric Early Rehabilitation. Robotics: Science and Systems; Workshop on Perception and Interaction Dynamics in Child-Robot Interaction (2017).

[20] Moritz Köster, Shoji Itakura, Masaki Omori, and Joscha Kärtner. 2019. From understanding others' needs to prosocial action: Motor and social abilities promote infants' helping. Developmental Science 22, 6 (2019), 1-8. https: //doi.org/10.1111/desc.12804
[21] Moritz Köster, Xenia Ohmer, Thanh D. Nguyen, and Joscha Kärtner. 2016. Infants Understand Others' Needs. Psychological Science 27, 4 (2016), 542-548.

[22] Do Kyeong Lee, Whitney G. Cole, Laura Golenia, and Karen E. Adolph. 2018. The cost of simplifying complex developmental phenomena: a new perspective on learning to walk. Developmental Science 21,4 (2018), 1-14. https://doi.org/10. $1111 /$ desc. 12615

[23] Ulf Liszkowski, Malinda Carpenter, and Michael Tomasello. 2008. Twelve-montholds communicate helpfully and appropriately for knowledgeable and ignorant partners. Cognition 108, 3 (2008), 732-739. https://doi.org/10.1016/j.cognition. 2008.06.013

[24] Dorothea U. Martin, Madeline I. MacIntyre, Conrad Perry, Georgia Clift, Sonja Pedell, and Jordy Kaufman. 2020. Young Children's Indiscriminate Helping Behavior Toward a Humanoid Robot. Frontiers in Psychology 11, February (2020), $1-13$.

[25] Dorothea Ulrike Martin, Conrad Perry, Madeline Isabel MacIntyre, Luisa Varcoe, Sonja Pedell, and Jordy Kaufman. 2020. Investigating the nature of children's altruism using a social humanoid robot. Computers in Human Behavior 104 (2020), 106149. https://doi.org/10.1016/j.chb.2019.09.025

[26] Catharine L R Mcghan, Ali Nasir, and Atkins Ella M. 2012. Human Intent Prediction Using Markov Decision Processes. Fournal of Aerospace Information Systems (2012), 1-16.

[27] Markus Paulus. 2014. The emergence of prosocial behavior: Why do infants and toddlers help, comfort, and share? Child Development Perspectives 8, 2 (2014), 77-81. https://doi.org/10.1111/cdep.12066

[28] Markus Paulus, Nina Kühn-Popp, Maria Licata, Beate Sodian, and Jörg Meinhardt. 2013. Neural correlates of prosocial behavior in infancy: Different neurophysiological mechanisms support the emergence of helping and comforting. NeuroImage 66 (2013), 522-530. https://doi.org/10.1016/j.neuroimage.2012.10.041

[29] Andreea Peca, Ramona Simut, Hoang Long Cao, and Bram Vanderborght. 2016. Do infants perceive the social robot Keepon as a communicative partner? Infant Behavior and Development 42 (2016), 157-167. https://doi.org/10.1016/j.infbeh. 2015.10.005

[30] Daniela Plesa Skwerer and Helen Tager-Flusberg. 2016. Empathic responsiveness and helping behaviours in young children with Williams syndrome. Fournal of Intellectual Disability Research 60, 10 (2016), 1010-1019.

[31] Laura A Prosser, Laurie B Ohlrich, Lindsey A Curatalo, Katharine E Alter, and Diane L Damiano. 2012. Feasibility and preliminary effectiveness of a novel mobility training intervention in infants and toddlers with cerebral palsy. Developmental neurorehabilitation 15, 4 (2012), 259-66.

[32] Stephanie Rosenthal, Joydeep Biswas, and Manuela Veloso. 2010. An effective personal mobile robot agent through symbiotic human-robot interaction. Proceedings of the International foint Conference on Autonomous Agents and Multiagent Systems, AAMAS 2 (2010), 915-922.

[33] Mark A. Schmuckler. 2001. What Is Ecological Validity? A Dimensional Analysis. Infancy 2, 4 (2001), 419-436.

[34] Nils Schuhmacher, Moritz Köster, and Joscha Kärtner. 2019. Modeling Prosocial Behavior Increases Helping in 16-Month-Olds. Child Development 90, 5 (2019), 1789-1801. https://doi.org/10.1111/cdev.13054

[35] Margarita Svetlova, Sara R. Nichols, and Celia A. Brownell. 2010. Toddlers' Prosocial Behavior: From Instrumental to Empathic to Altruistic Helping. Child Development 81, 6 (2010), 1814-1827. https://doi.org/10.1111/j.1467-8624.2010. 01512.x

[36] Felix Warneken and Michael Tomasello. 2007. Helping and cooperation at 14 months of age. Infancy 11, 3 (2007), 271-294. https://doi.org/10.1111/j.15327078.2007.tb00227.x

[37] Felix Warneken and Michael Tomasello. 2008. Extrinsic Rewards Undermine Altruistic Tendencies in 20-Month-Olds. Developmental Psychology 44, 6 (2008), 1785-1788. https://doi.org/10.1037/a0013860

[38] Felix Warneken and Michael Tomasello. 2009. Varieties of altruism in children and chimpanzees. Trends in Cognitive Sciences 13, 9 (2009), 397-402. https: //doi.org/10.1016/j.tics.2009.06.008

[39] Yuuki Yasumatsu, Taichi Sono, Komei Hasegawa, and Michita Imai. 2017. I can help you: Altruistic behaviors from children towards a robot at a kindergarten. In Companion of the ACM/IEEE International Conference on Human-Robot Interaction. 331-332. https://doi.org/10.1145/3029798.3038305

[40] Ashkan Zehfroosh, Elena Kokkoni, Herbert G Tanner, and Jeffrey Heinz. 2017. Learning models of Human-Robot Interaction from small data. In Proceedings of the 25th Mediterranean Conference on Control and Automation. 223-228.

[41] Ashkan Zehfroosh and Herbert G Tanner. 2019. Reactive motion planning for temporal logic tasks without workspace discretization. In Proceedings of the IEEE American Control Conference (2019).

[42] Ashkan Zehfroosh, Herbert G Tanner, and Jeffrey. Heinz. 2018. Learning option MDPs from small data. In Proceedings of the IEEE American Control Conference (2018). 